\title{
loT-enabled dynamic lean control mechanism for typical production systems
}

Kai Zhang, Ting Qu, Dajian Zhou, Matthias Thurer, Yang Liu, Duxian Nie, Congdong Li and George Q. Huang

The self-archived postprint version of this journal article is available at Linköping University Institutional Repository (DiVA):

http:// urn.kb.se/ resolve?urn=urn:nbn:se:liu:diva- 155560

N.B.: When citing this work, cite the original publication.

The original publication is available at www.springerlink.com:

Zhang, K., Qu, T., Zhou, D., Thurer, M., Liu, Y., Nie, D., Li, C., Huang, G. Q., (2019), IoT-enabled dynamic lean control mechanism for typical production systems, J ournal of Ambient Intelligence and Humanized Computing, 10(3), 1009-1023.

https:// doi.org/ 10.1007/ s12652-018-1012-z

Original publication available at:

https:/ doi.org/ 10.1007/ s12652-018-1012-z

Copyright: Springer (part of Springer Nature) (Springer Open Choice Hybrid J ournals)

http:// www.springer.com/gp/products/journals 


\section{IoT-enabled dynamic lean control mechanism for typical production systems}

Kai Zhang, 1,3

Ting Qu, 2曰,3

Email quting@jnu.edu.cn

Dajian Zhou, 4

Matthias Thürer, 2,3

Yang Liu, 3,5

Duxian Nie, 6

Congdong Li, 1,3

George Q. Huang, 3,7

1 School of Management, Jinan University, Guangzhou, 510632 China

2 School of Electrical and Information Engineering, Jinan University (Zhuhai Campus), Zhuhai, 519070 China

3 Institute of Physical Internet, Jinan University (Zhuhai

Campus), Zhuhai, 519070 China

4 Guangdong CIMS Provincial Key Lab, Guangdong University of Technology, Guangzhou, 510006 China

5 Department of Management and Engineering, Linköping University, 581 83 Linköping, Sweden

6 College of Mathematics and Informatics, South China Agricultural University, Guangzhou, 510642 China

7 Department of Industrial and Manufacturing Systems Engineering, The University of Hong Kong, Hong Kong, China

Received: 27 April 2018 / Accepted: 24 August 2018 


\section{Abstract}

The emergence and subsequent popularization of lean has been one of the most significant developments in the history of operations management. However, there is a lack of systematic theory on the control framework underlying lean production. It is therefore difficult to conduct more in-depth research on Lean theory, specifically in the context of emerging technologies as smart manufacturing or Industry 4.0. In this study, process control theory is used to re-define several major lean methods and tools. Then a Lean-Oriented Optimum-State Control Theory (L-OSCT) is proposed that integrates these lean methods and tools into optimum-state control theory. On the level of method and mechanism, we adopt a recently emerged synchronization approach to obtain global-wide leanness of a large-scale system. L-OSCT provides dynamic process control in industrial networking systems. At last, a case study in a large-size paint making company in China is used to validate the effectiveness of the approach.

AQ1

\section{Keywords}

Lean production

Process control

Internet of things

Just in time

Customized production

\section{Introduction}

Lean production (LP) is a production management concept that takes customer demand as major driving force while pursuing minimum capital investment cost and maximum operation efficiency through continuous improvement practices and waste elimination (Shah and Ward 2003, 2007; Holweg 2007). LP is a multidimensional approach which encompasses a wide variety of management practices - including Just-in-Time (JIT), visual management, Kanban management, cellular manufacturing, supplier management, etc.-in an integrated system (Ohno 1988; Riezebos et al. 2009; Sugimori et al. 1977; Holweg 2007). The general essence of LP is to transition from the actual state of a production resource, a production process or a production system to an ideal state which meets the most preferable demands. 
Since its inception a variety of lean methods, tools and practices have emerged, reflecting that different enterprises have different goals and different development stages for achieving lean (McLachlin 1997; Jasti and Kodali 2016). At the same time, there has not been a complete, systematic and feasible theory to define and describe the core management essence and the underlying control framework of LP. This makes it not only difficult to conduct more in-depth research on LP theory in the context of next-generation production modes, such as smart manufacturing or Industry 4.0, but also challenges lean practitioners when they seek to identify suitable lean method for their specific problems.

Herron and Braiden (2006) defined three stages on a firm's journey towards LP. The first stage is an operation-based LP stage which aims to stabilize the manufacturing system to ensure that processes are under control and reliable. The second stage is a plan-based LP stage which aims to achieve maximum productivity through the application of process-based LP management tools such as JIT production. The last stage is an incremental stage to realize further LP improvement in other situations. Based on this categorization, this paper will further investigate the control essence of lean practices in the context of newly emerging realities in terms of demand requirements and technical possibilities to help fulfill this demand. In terms of demand, we observe a transition from repetitive orders, mass production mode with stable production processes to customized demands, small-batch multi-variety production mode with highly dynamic production processes (Sim and Rogers 2008; Fogliatt et al. 2012; Deshmukh et al. 2017). In terms of technology available to help fulfill this demand, production execution is transitioning from manual operations to automatic and even intelligent operations, the production data is transitioning from closed and backward to real-time and transparent data, and production planning is transitioning from prior-plan mode to adaptive decision making and online control (Powell and Skjelstad 2012; Olivella et al. 2008; Zhang et al. 2015; Marodin and Saurin 2013). The emergence of these new backgrounds motivates us to unify LP through re-defining the control logics of traditional LP methods using a process control perspective, e.g. lean objective, management level, undertaking object, improvement means, evaluation standard, etc.

The primary aim of this paper is to investigate how the Optimum-State Control Theory (OSCT), which is proposed by the authors' team, can be extended to systematically integrate the various LP methods and tools into a unified and extensible theoretical framework designated as Lean-oriented Optimum-State Control Theory (L-OSCT). More specifically, three questions are of interest to the development of L-OSCT. First, what is the management or control essence of the various LP methods and tools, and how to understand, depict and model the 
various LP methods within a consistent lean control system? Second, how to extend the OSCT to realize L-OSCT so as to deal with the newly emerged lean control demands from contemporary production systems such as dynamic demands and lean measurements, high responsiveness and adaptability, selfmanaged decision-making and control ability. Third, how to define and obtain the overall optimal lean objectives and synchronously control multiple subsystems to their lean-state in the context of large-scale dynamic production systems composed of multiple workshops or even factories? Answering these questions, we will develop L-OSCT. A short case is then presented to provide a first assessment of its applicability.

The remainder of this paper is organized as follows: Sect. 2 briefly introduces the basic of OSCT and describes the extension proposed in this study: L-OSCT. Section 3 proposes the dynamic control mechanism for L-OSCT focusing on two typical production systems. Section 4 then explains how to use L-OSCT for both types of typical production systems before a case study to test the effectiveness of L-OSCT is presented in Sect. 5. Final conclusions are summarized in Sect. 6.

\section{Lean- oriented optimum-state control theory}

\subsection{Lean control framework}

Figure 1 is the lean control framework which supported by approaches and mechanism. The approaches are including IoT based Synchronization, Multidisciplinary Design Optimization (MDO) and so on. And the optimumstate control theory is the is the supporting mechanism. The lean level consisting of three main levels: The first level of lean practice is operation-oriented lean. Due to the simplicity and repeatability of production operation, the major works of lean improvement focus on monitoring and correcting the non-standard operations of workers (Huang et al. 2008; Brewer et al. 1999; Qu and Huang 2012; Chongwatpol and Sharda 2013). The lean objective of this level has been fixed as a set of long-term operation standards which ensure that the performance expectation of an individual production resource or cell is efficient and stable. The second level of lean practice is process-oriented lean $(\mathrm{Qu}$ and Thürer et al. 2017; Chow et al. 2006). Due to the graduate maturation of lean field operations skills as well as the improvement of mechanization levels, the operation performance of each production resource can be considered sufficiently lean. The major focus of further lean improvement will be the pursuit of the continuity of the production process through minimizing the difference between adjacent resources. The lean objective of this level comes from a short-term, e.g. daily, production plan which helps achieving maximum productivity of a production process. The third level of lean practice is demand- 
oriented lean. Unlike the first two levels which have clear lean objectives, either as operation standards or production plan, this level does not have a definite lean objective. This commonly occurs when a production process has to deal with a high variety of customized production orders. The lean objective of this level is determined dynamically during the execution of a production process. The first level ion our framework refers to the operation-oriented primary lean practices which heavily involves manual works, while the other two levels refer to the process-oriented advanced lean practices which mainly depend on the ability of computing and intelligence.

\section{Fig. 1}

Lean control framework

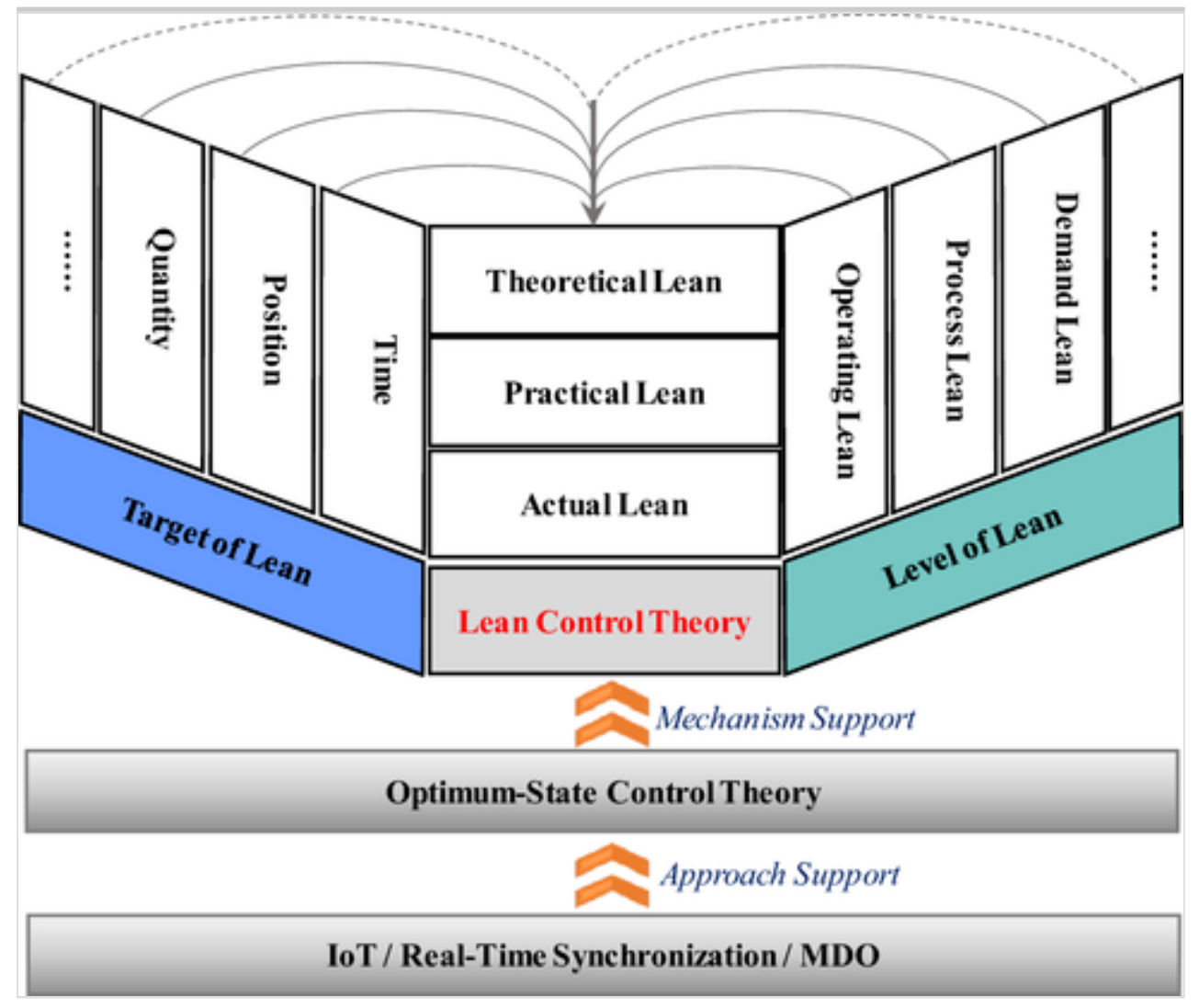

Each manufacturer in practice will take a specific LP method or tool to pursue one or several idiosyncratic key performance indicators. The most common lean practice is JIT, which is time-oriented and seeks to realize delivery of a specific material or product as close as possible to its actual demand. Other lean methods include zero-inventory management, which is inventory-oriented and seeks to minimize the WIP inventories in the production processes, and zero-defect management which is quality-oriented and aims at the reduction of defects through prevention etc. Time, inventory and quality-objective are referred to as 
the lean objectives of a production system (Qu and Luo et al. 2012; McLachlin 1997) in this paper.

A production system comprises both: technological elements and organizational behavior. Technological elements are the resources while the organizational behavior is usually part of lean process control. The recourse layer usually includes hardware equipment, software equipment, workers and so on, while the lean process control layer makes decision according to scientific decisionmaking technology and control methods to control the optimization of production operations. The production system contains one or more independent decision units, each of which has this two-tier structure.

Recently, in the background of Industry 4.0, Advanced Manufacturing Partnership (AMP) or Made in China 2025, production systems are equipped with more automated, and even flexible equipment and methods. But with the increasingly diverse and personalized requirements of customers, the production process is subject to increased dynamics. This requires production resources that are flexible enough to accommodate the increasing impact of dynamic demand. The production system should be capable to identify interferences in the production process rapidly and accurately and then take measures to regain control of the operating state of the system under the established lean indicators, i.e. the Optimum-State. This control philosophy is called Lean-Oriented Optimum-State Control Theory (L-OSCT).

\subsection{Challenges in lean-oriented optimum-state control}

L-OSCT is designed to provide a solution for the entire production system. There are three challenges in the realization of this objective:

- Incomplete acquisition and processing of information from technological elements: The data types and dimension are complicated due to the complex technological elements in the production environment. Multiple data acquisition methods are used to collect data and there is lack of standards for data collecting and transfer. All these reasons cause difficulties in information acquisition and processing.

- Lack of decision making capability: Due to the lack of a real-time information execution layer and scientific techniques or methods, decisionmaking relies on experience. As a result, decision-making lags behind process execution and cannot be implemented in accordance with the actual situation to make timely and effective adjustment. 
- Sub-optimization: The complex production system is composed of multiple independent decision-making units, but the overall Optimum-State of the system is not linearly superimposed by the lean indicators of the independent decision-making units.

\subsection{Optimum-state control theory}

Optimum-State Control Theory (OSCT) is a control theory that can be used to make adaptive decisions to regain control after the system was affected by dynamics, so that the system again operates in the Optimum-State. Figure 2 schematizes OSCT.

\section{Fig. 2}

Optimum-state control theory

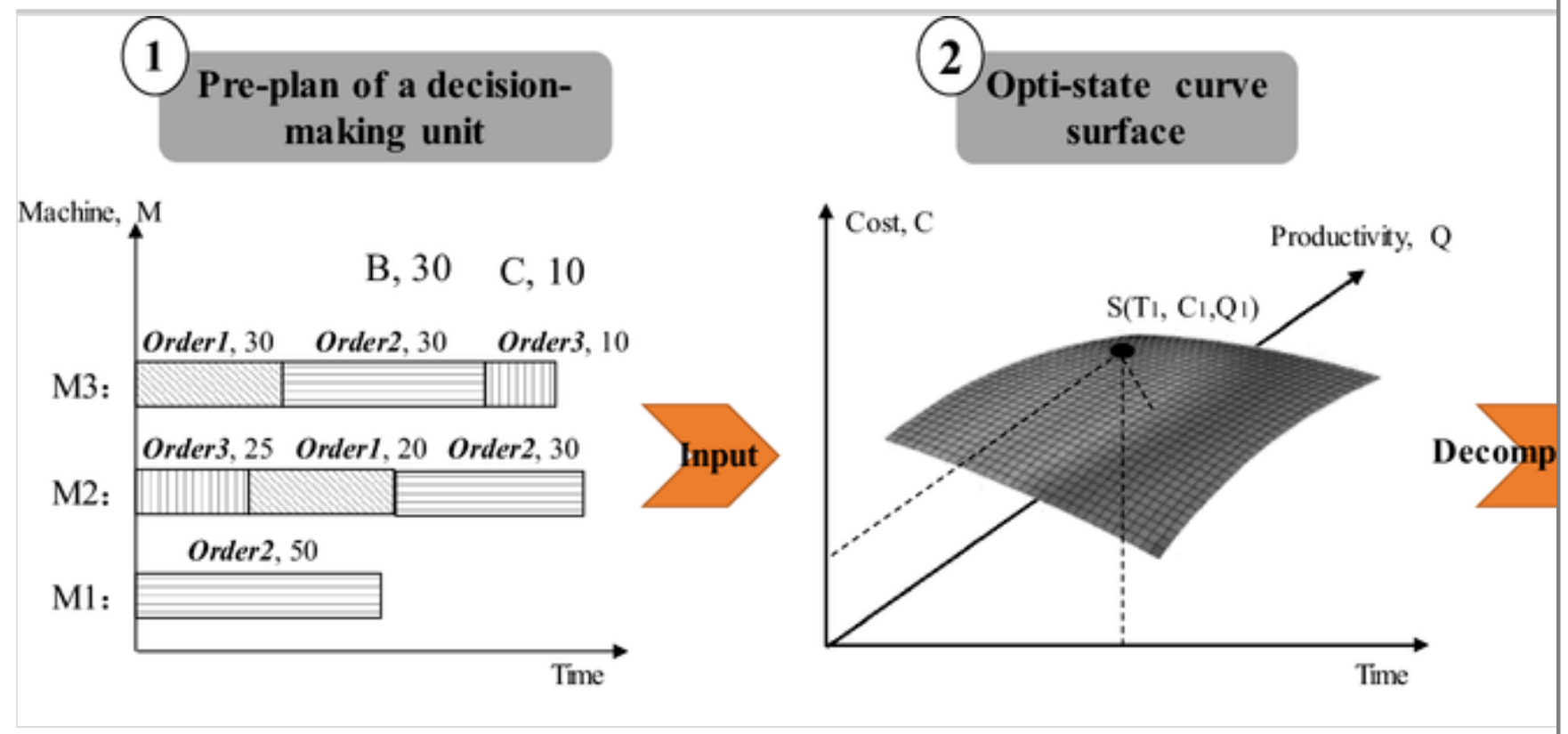

Figure 2 shows the three main parts of OSCT: Pre-plan of a decision unit, optistate curve surface and opti-state curve (Qu and Zhang 2018). The opti-state curve is the core of OSCT. It supports the determination of optimum decisions at the execution layer. The opti-state curve surface represents the whole optimization goals of one production system and the surface can dissolve into different curves for each target, which is controlled by the opti-state curve. The Pre-plan of a decision unit is the input of the opti-state curve surface. The production system consists of two Optimum-States in OSCT: Theoretical Optimum-State and Practical Optimum-State.

- The theoretical optimum-state: is the mapping of the actual requirement, 
which is made by the production execution layer in combination with the actual requirements and the configuration of resources, the state of execution to make the state (part 1 of Fig. 2). Theoretical Optimum-State is usually before production, for example, the production schedule Gantt-chart is a Theoretical Optimum-State.

- The practical optimum-state: is the corrected status of the Theoretical Optimum-State. The Practical Optimum-State is made by the decisionmaking layer in response to dynamic impacts on the system and the realtime status of resources. The Theoretical Optimum-State can no longer be used to guide the execution after the production process has been disturbed. The Practical Optimum-State is based on the real-time status of the production system, so the Practical Optimum-State enables more precise decision making.

Since the production system pursues different dimensions of the optimal state at the same time, such as the shortest time, the lowest energy consumption and so on, OSCT also controls multi-dimensions Optimum-State at the same time, e.g., time dimension Optimum-state, energy dimension Optimum-state. These states coupled to each other together constitute an Optimum-State curved surface (part 2 of Fig. 2).

For the single-dimensional Optimum-State, the actual state deviates from the Theoretical Optimum-State when the dynamic occurs. In the absence of any measures to respond, the actual state shifts in a non-optimal operating state, and the difference between Theoretical Optimum-State and actual keeps increasing. When the deviation reaches a certain threshold, the system triggers the optimumstate control: The decision-making layer calculates the Practical Optimum-State of operation in line with the current state obtained from real-time field data and then guides the executive layer to run counter-measures in accordance with the state (part 3 of Fig. 2).

\subsection{OSCT for lean production system}

OSCT is integrated into LP in this study to create L-OSCT. The curve of LOSCT is shown in Fig. 3 (Qu and Zhang 2018). A theoretical lean curve is sued to guide the implementation of lean at production. After production starts, the execution layer is progressively carried out in accordance with the theoretical lean curve. When a dynamic disturbance, such as order changes, machine breakdown, causes the actual lean curve to deviate from the theoretical lean curve (as point A) above a certain threshold, response measures are triggered by L-OSCT to regain control over the production system (as point B). 
Fig. 3

L-OSCT and lean control triangle

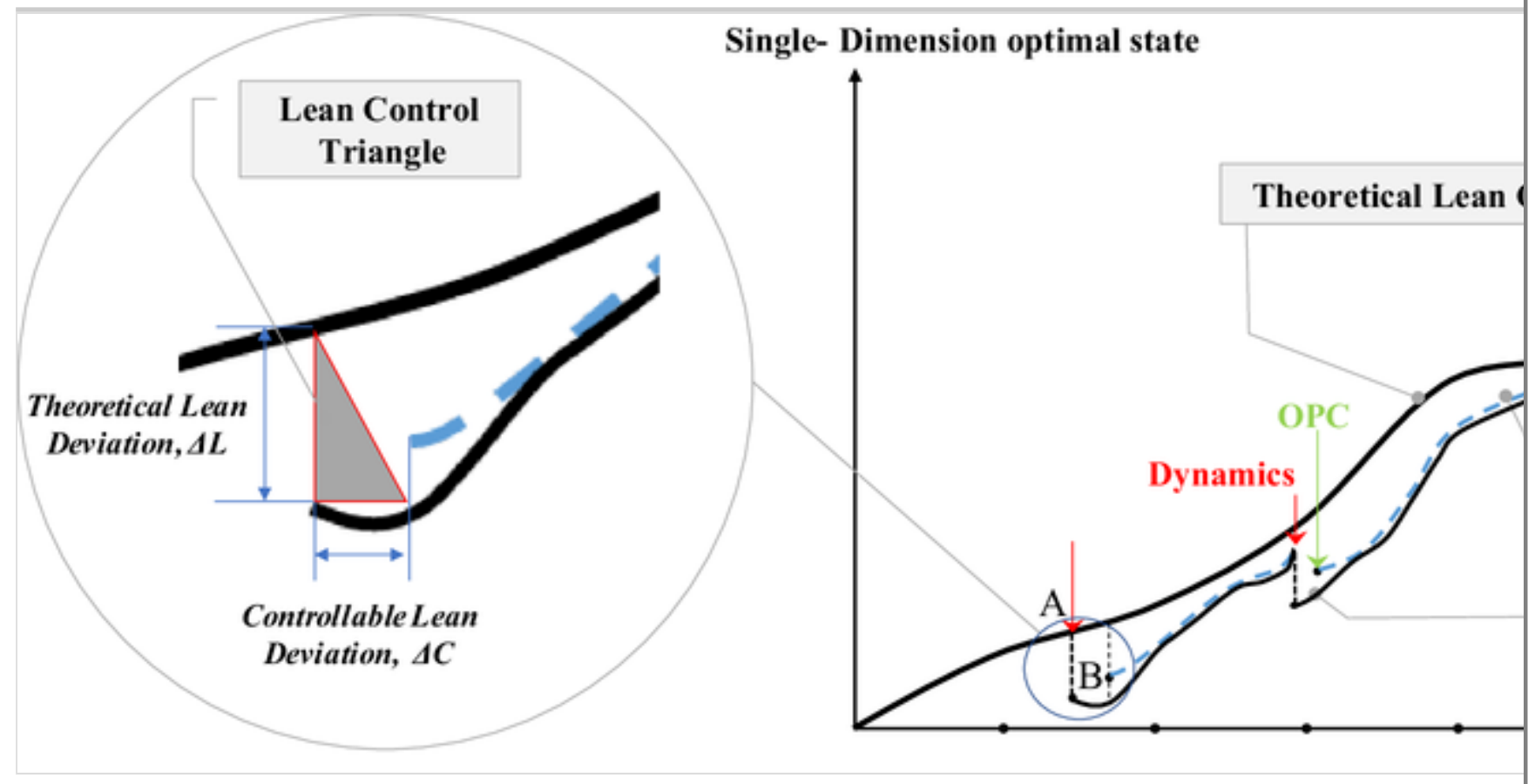

At this point, according to the implementation of the production system realtime status, the decision-making layer calculate a new situation in time and accurately in line with the current lean curve, here called the practical lean curve. As the execution state has changed, the theoretical lean curve provides no longer guidance for the subsequent operation of the system. Rather the optimal operating state of the system should be run with the practical lean curve. After the practical lean curve is obtained, the execution state is approaching the curve quickly and the system returns to the Optimum-State. The process is cycled through the production process until the end of production.

The zoom part of Fig. 3 shows the so-called Lean Control Triangle. The two sides of this triangle are important for L-OSCT since they represent:

- Theoretical lean deviation: The theoretical lean deviation is the difference between the theoretical lean curve and the practical lean curve, marked as $\Delta \_l$. It is due to the impact of dynamic on the system and its size is determined by system configuration and environmental dynamic.

- Controllable lean deviation: The controllable lean deviation is the response time of L-OSCT in terms of calculation of the new practical lean curve and indicators, marked as $\Delta_{-} c$. It reflects the efficiency of the response to the dynamic. 
The "lean control triangle" area consequently reflects the performance of LOSCT; the purpose of lean control is to reduce the area of the triangle. It is easy to see that reducing the triangular area can be achieved by two ways - to reduce the theoretical lean deviation and to reduce the controllable lean deviation.

The theoretical lean deviation reflects the robustness of the system. The higher the robustness, the less the influence of the dynamic on the system. The optimal configuration of production resources is an effective way to improve the robustness of the system. The controllable lean deviation reflects the efficiency of L-OSCT response to the dynamic. The response performance of OSCT is determined by two aspects: real-time and sufficient information from the execution layer and efficient control mechanism.

\section{Dynamic control mechanism of L-OSCT}

A production system can be composed of a single decision-making unit or a plurality of decision-making units. For the multi-units production system, the Optimum-State (local lean) coupling of each subsystem forms the OptimumState of the whole large-scale system (global lean). Global lean is not simply linear superposition of local lean, and the Optimum-State of each subsystem requires a fine control model or mechanism for collaborative optimization. On the other hand, for the single-unit production system, the Optimum-State of the system is the Optimum-State of the unit. This section will propose a L-OSCT control mechanism based on theoretical and actual lean curve for each of the two systems.

\subsection{Information framework for the dynamic control mechanism}

The basis for using L-OSCT is that the real-time information from the execution layer of the production system should be collected first. The Internet of Things (IoT) is used to build the information environment in this study. For the control mechanism synchronization theory is used to make an Optimum-State decision.

The dynamic control mechanism is based on the construction of a reasonable information framework. AUTOM, which structure is depicted in Fig. 4 below, is used as the basic structure (Huang and Qu 2012a, b; Huang and Qu 2011).

\section{Fig. 4}

Information framework for the dynamic control mechanism 


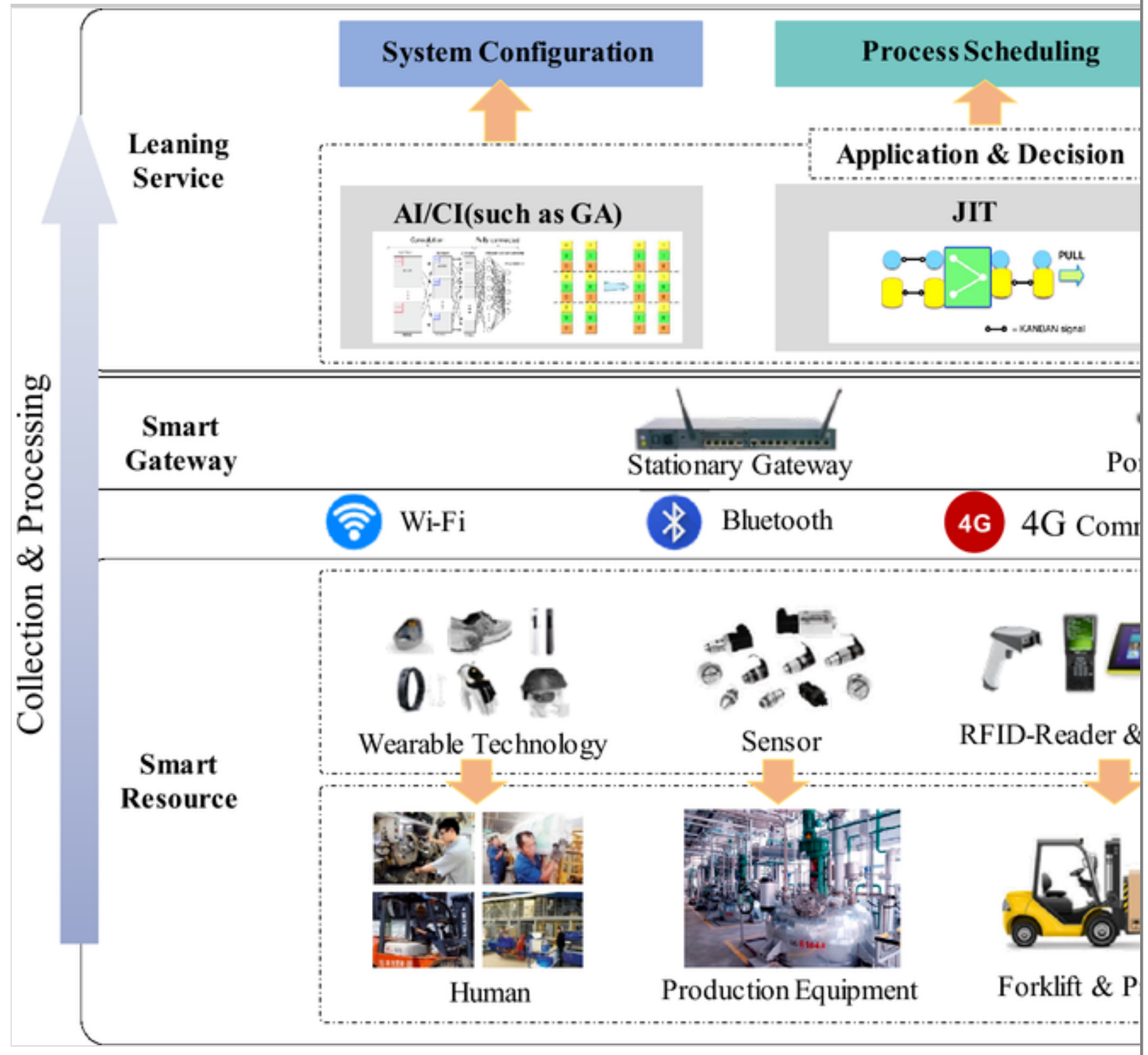

There are three main part of this information framework:

Smart resources: all the physical resources are in this layer, which contains plants, warehouses, workers, machines and so on. Physical resources are provided with suitable IoT devices transforming them into smart recourse. The IoT devices includes: RFID tags, readers, bar code, tablet, wearable devices and so on. These smart resources also include cloud resources.

Smart gateway: the main function of this layer is to gather information and exchange real-time data. It is the information hub of the system and it connects the enterprise information system and the decision-making system.

Lean service: to provide lean services such as lean manufacturing scheduling, lean system configuration, etc. for the production systems various types of 
scientific decision-making and control methods, such as lean tools, AI/CI, synergy theory and so on are used.

\subsection{Dynamic control mechanism for single-unit production system}

To achieve the Optimum-state for single-system production system a preplanned plus a dynamic planning approach are used. Pre-planned is the plan made by the system before production according to the requirements and resources configuration. Dynamic planning is the plan made when the dynamics occurs with the guidance of the Lean Control Triangle using mature scientific decision-making methods, such as artificial intelligence, optimization algorithm and so on.

\subsection{Dynamic control mechanism for multi -unit production system}

For this kind of system, the synchronization theory is referred to make an Optimum-State decision as the control mechanism (Qu and Lei 2016; Qu and Pan 2017a, b).

\subsubsection{The level of synchronization}

The production system consists of several independent decision-making units, each unit has its capacity range to respond to the impact, which is marked as $\mathrm{R}$ [min, max]. Meanwhile the dynamic influence range is marked as D.

The organizational behavior layer of the production system will make a predecision after it gets the requirements, then the production system executes the plan. Once the execution is subject to dynamics, there will be three degrees of response to deal with the impact of dynamics:

- Internal re-plan synchronization: the dynamic impact is not large. The affected units do not need to introduce new resources and the impact can be eliminated by re-planning. At this time $R_{\min }<D<R_{\max }$.

- External re-plan synchronization: When the capacity of existing resources within the affected unit cannot cope with the dynamic impact, the remaining units associated with it need to coordinate decisions and re-plan to eliminate the impact of the dynamics on the production system. At this time, $D>$ $R_{\max }$.

- Requirement synchronization: Regardless of how the production system 
allocates internal resources and even the introduction of external resources, it cannot deal with the dynamic impact, which requires synchronization from the demand side, for example, whether the customer can change the delivery date. At this time, $D>\mathrm{RS}_{\max }\left(\mathrm{RS}_{\max }\right.$ is the max ability of the production system to respond to the impact).

\subsubsection{Production system synchronization}

The IoT-enabled information framework provides real-time data support for lean control. On the other hand, an effective synchronization approach is needed to synchronize all the decision-making units in the production system to deal with the dynamic impact in order to calculate the practical lean indicators and then guide the execution. Figure 5 shows the control flow of this response mechanism.

\section{Fig. 5}

Mechanism process of dynamic control 


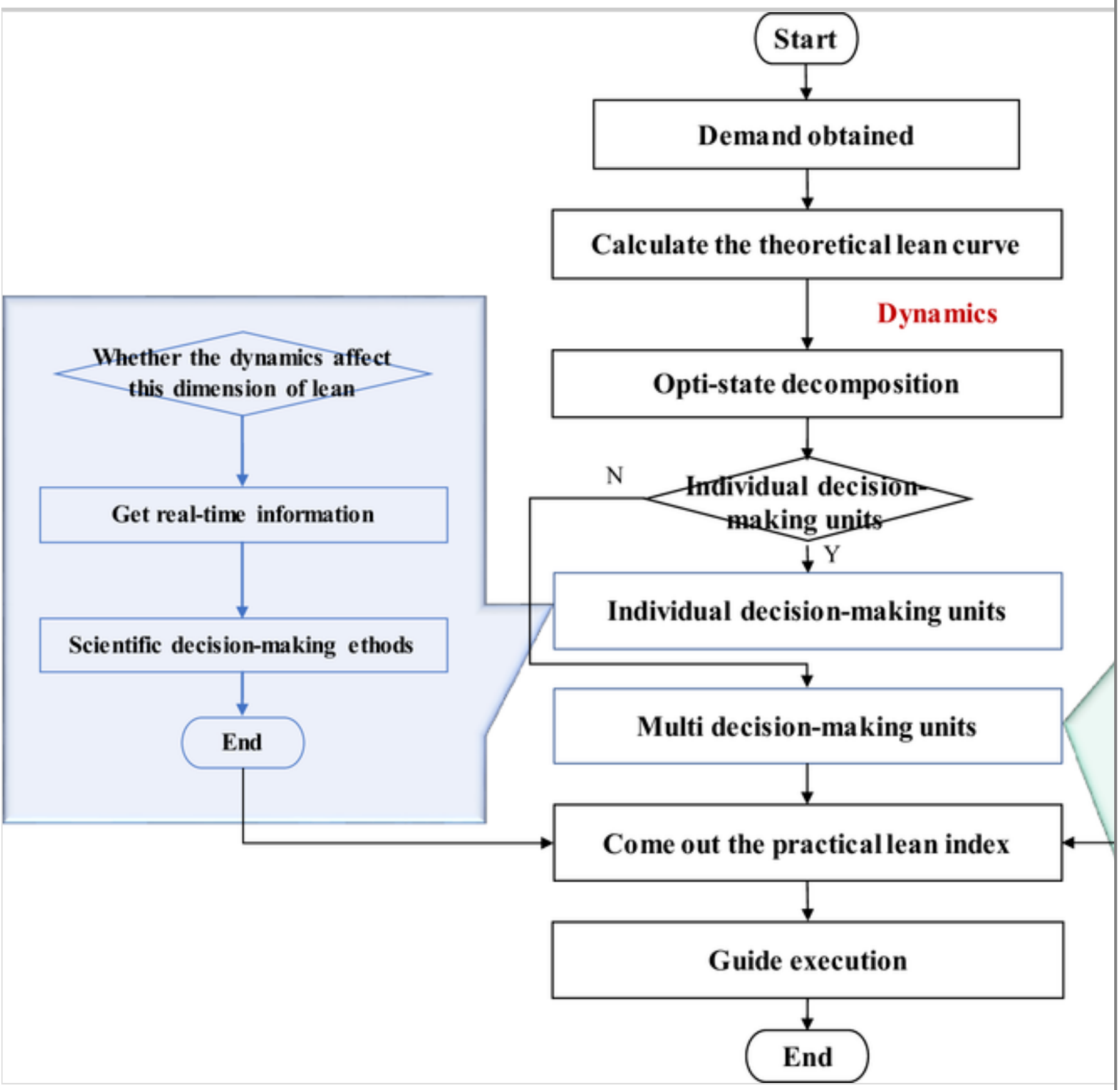

The workflow of the dynamic control mechanism is as follows:

Step1: Production system obtains the requirements;

Step2: Calculate the theoretical lean curved surface for the system based on the requirements and the configuration of system, then make pre-plan for each unit, such as production plan;

Step3: The system was affected by dynamics, which cause the execution state and the lean curve to deviate;

Step4: Decompose the multi-dimension optimum-state for lean production system; 
Step5: Take a different control mechanism according to whether the system is a single decision-making unit or multi-decision unit:

- Option 1: For single decision-making unit system

a. Decomposition of multi-dimensional optimum-states to determine the dynamic impact on those optimal target;

b. Get real-time information on the execution layer;

c. Make an optimal decision using mature scientific decision-making methods, e.g., artificial intelligence, optimization algorithm.

- Option 2: For multi decision-making unit system

a. Decomposition of multi-dimensional optimum-states to determine the dynamic impact on those optimal target;

b. Get real-time information on the execution layer;

c. Determine the scope of the dynamic impact and the scope of coping ability of every unit, If $R_{\min }<D<R_{\max }$, use internal re-plan synchronization;

d. If $D>R_{\max }$, use external re-plan synchronization;

e. If $D>\mathrm{RS}_{\max }$, use requirement synchronization;

f. Solve the problem from the perspective of theoretical lean difference, by changing the robustness of the system to enhance the ability of the system to resist the impact of dynamic, if the control lean cannot eliminate the influence of dynamic;

g. After the dynamic lean control, draw the practical lean curve to instructs the execution layer to execute;

h. End the dynamic lean control mechanism.

\section{Dynamic lean control on typical production systems}

This section explains how to use the L-OSCT for both types of typical production systems. 


\subsection{L-OSCT on single-unit production system}

The application of L-OSCT is introduced from two typical independent decisionmaking units which are production unit and the warehouse unit.

\subsubsection{IoT-enabled production line management system}

The intermittent accumulation of products between processes and the intermittent waiting between processes will result in longer manufacturing cycles and difficulty in making full use of resources. Just-in-time is usually used to improve such situation in traditional lean production, using Kanban to link supply, production and sales closely to reduce the inventory cost, product store and WIP, while improving production efficiency. An important feature of JIT is that everything should be strict to the plan, however, with the uncertainty and variety of customer requirements, pre-planned usually face a large number of uncontrolled dynamics, which leads to the situation where the plan (theoretical lean) cannot adapt to the actual production situation.

WIP can affect various areas in the manufacturing process such as productivity and line efficiency. This is especially important for bottlenecks in a manufacturing operation. WIP control is one of the most important parts in production line management. So, L-OSCT on production line management system should be focus on the WIP control.

To start with, all necessary production resources such as machines, pallets, buffer areas, employees should receive RFID tags and readers or other IoT devices. After that the shopfloor transforms into a IoT environment. A typical discrete manufacturing company plans and controls the overall production process through a production manager. With L-OSCT the system could get the lean control by reducing the controllable lean deviation. Before dynamic lean control, the capacity of the machine tools, production lines and personnel should be modeled. When a worker log in the system, he/she just need to work following the plan according to the real-time production operation. The WIP that accumulated before and after the assembly workstation will be uploaded to the system in real-time, so that the system automatically generates the transport task, and arranges the transportation unit to carry out the process of the transportation, to ensure the production system has high fluency. When the dynamic happens on the production plan (such as new orders added) or the production site (such as the damage of equipment), the system and the administrator will determine required tasks and push the tasks to the corresponding working unit in time.

With the L-OSCT, the system has a high property of lean, there are three main reasons: 
1. It reduces the waste caused by the invalid of the material, the unbalance operation, and the inappropriate plan.

2. When there is a dynamic impact, the system can make a timely decision to modify the arrangement of the units to respond.

3. It improves the flow of the production system in a dynamic environment.

\subsubsection{Warehouse management system based on Internet of Things}

In the warehousing process, the transportation management of raw materials, semi-finished products and finished products will cause wastes of time, place and human resources due to the unbalanced job arrangement, improper planning and other events caused by the placement and accumulation. In recent years, and with increasing diversity in customer demands, using the traditional lean tools such as on-site management, manual plan correction or elementary information technology to manage the data may cause a series of problems like low accuracy rate, or high delay rate. The RFID electronic tags related technology could shorten the operation time and reduce the occurrence of the above problems.

In the key aspects of warehouse management, such as location allocation, route planning and so on, L-OSCT can help the decision layer make a scientific decision guiding the execution layer. This includes:

1. Improved warehouse management correctness, management accuracy and ease of operation to reduce waste in inventory.

2. Improve operational efficiency of warehousing with the aid of RFID technology, attached electronic tags on goods packaging, cargo space and pallets to manage warehouse.

3. Improved accuracy of entry, exit, deposit and shift data, reduces the dependence on people in daily work reducing the risk of human error.

\subsection{L-OSCT on multi-unit production system}

The operation of the production system is made up of several subsystems. In the company, these subsystems are often some individual departments, or even some different cooperative companies. Due to the disconnection of information between subsystems and lack of association of business logic, it is difficult to achieve global optimization. Consequently, there have been a series of problems, 
such as insufficient or accumulation of production caused by the nonsynchronization of material supply and production, accumulation or spare of the warehouse caused by the non-synchronization of warehousing and production, and other problems caused by non-synchronization among subsystems. Because that every subsystem shares the real-time information, once a subsystem made an active decision-making adjustment due to the dynamics other related subsystem will respond to this adjustment making a passive decision. In order to achieve the overall effect of lean, every subsystem executes the plan in a synchronized way, which is how the L-OSCT works and all subsystems could be in a dynamic synchronization mode with L-OSCT.

The two key subsystems of a production system are taken as the example: the production system and logistics system. For the range of logistics, logistics system includes two subsystems which are warehouse and transporting. So, in the lean production system, production logistics synchronization means the synchronization between production, warehouse and transportation. The core of the lean control system is using L-OSCT to coordinate the three subsystems effectively.

In traditional production and logistics processes there is no sharing of real-time information between production and warehousing, and the decision of these two subsystems is not synchronized, so it will cause a series of problems. Using the traditional Kanban management and manual methods is only waiting negatively, resulting in a lot of waste, so it is inefficient for the departments in coordination under a high dynamic situation. The L-OSCT effectively synchronizes production and logistics by joint configuration of resource and determination of operation plan and the control parameters, to pursue the intensification of resources and the optimization of the overall operation.

By attaching IoT devices on products, pallets, shelves and forklifts, and configure the reader for the production nodes, warehouses, worker, the system can access to the real-time information of every element. When the system is affected by dynamics, the decision-making layer of production and logistics can control and supervise the overall plan using the real-time data collected from the executive layer and L-OSCT. After the decision-making, the plan is split into different subdivision plans and sent to the corresponding execution unit by LOSCT and pushed to the units for execution adjustment.

With the L-OSCT, the Lean Production System has a high property of lean which include: 
the real-time requirement of production, and reduce unnecessary stock out tasks.

2. Synchronized finished product warehousing. The warehouse can make a global optimal stock plan according to the inventory situation and the production schedule plan, and so does the production.

3. Synchronized production and WIP monitoring, which enhance the flow of the production process.

\section{Case study}

Combined with the application theory of L-OSCT in the production system, several systems running for some company have already been developed and implemented by the authors' team. In order to avoid repetitive discussions, one large-scale synchronization system is taken as the case.

\subsection{About the case company}

The project cooperation company is a large paint manufacturer, ranking top 50 at the same industry in the world and producing a wide range of paint products. After years of development, the company has achieved operating lean and process lean control. In term of operating lean control, the $5 \mathrm{~S}$ and Six Sigma has adopted; at the aspect of process lean, the company has invested heavily in building a company information system. At the planning level, the production plan is mainly based on ERP and other information systems. In the field of execution, the production tasks are mainly done manually based on paper records. In recent years, there has been increased uncertainty in demand. In addition, demand became more customized, which results in large dynamic in the production plant. At the same time, as limited warehouse resources, the case of warehouse explosion is unavoidable. As a result, relying solely on operating lean and process lean control methods has been unable to meet production needs, resulting in a situation in which production plans cannot be accurately implemented. The author's team have developed the third level lean (demand lean) IOT system for this company. This section will show the effect of the application of L-OSCT.

\subsection{Problem description}

\subsubsection{Production logistics operation process}

The company produces to-order. There are seven production workshops and two finished goods warehouses, which are respectively used for the production and 
storage of different types of products in the company. Typically, a customer's order contains multiple products, each of which can only be completed in one workshop. All types of products for each order must be shipped after they have been produced and shipped to the warehouse. The production process is shown in Fig. 6. When receiving a customer orders, customer service assigns production tasks to the workshops three days before the due date of the order considering the workshop operation status. Then the workshops make production scheduling according to the actual operation state. Meanwhile, the warehouse receives the delivery tasks and makes storage allocation and delivery planning according to the actual situation of the warehouse the day before the orders are delivered to the customers. The production scheduling plan, the allocation plan and other plans are all theoretical lean decisions.

Fig. 6

Production-logistics operation process

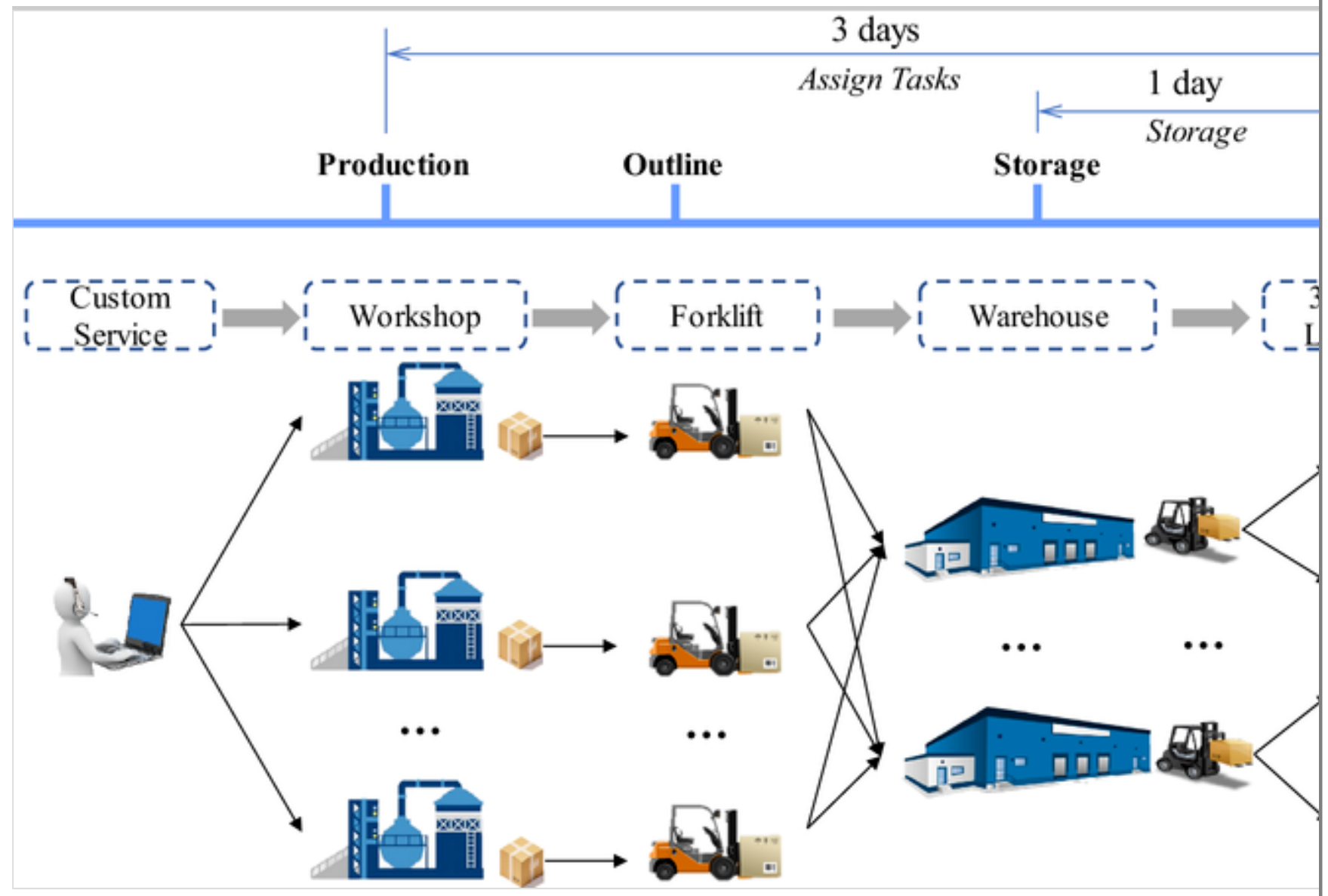

\subsubsection{Problems in the production logistics operation}

The following main issues could be encountered with the current control system:

(i) Manual recording methods are still in use, for instance, filling the storage information on the Kanban at the entrance of the warehouse. (ii) Due to the lack 
of real time information, it is difficult for the decision maker to develop lean plans combining the real-time status of the production field; (iii) in the actual production operation environment exists huge stochastic dynamic. It lacks realtime information about the production logistics process and scientific and effective decision control mechanism for correcting the plan to allow the system to achieve the demand lean when the actual execution state deviates from the theoretical lean, which may result from the delay of the tasks; (iv) The workshop subsystem and the storage subsystem make the decision separately and there is a lack of real-time information sharing. When a system is out of execution, it's unable to coordinate the global resources to make the global optimal theoretical lean decision.

\subsection{IoT-based environment deployment}

In order to solve the problems identified above, a real-time production logistics management system based on IoT was developed. Its main function lies in two aspects: on the one hand, in response to the problem of untimely information collecting, un-lean planning and inaccurate executing, IoT-based technology and intelligent decision-making methods are utilized to realize accurate pre-planning in different departments of production logistics. On the other hand, in response to the dynamics of the production process and the inability of effective cooperation between production and warehouse system, L-OSCT is used to synchronize the decision of production and logistics. In the implementation process, when the dynamic occurs, the system rapidly makes a lean decision to re-generate the practical lean curve. Meanwhile, it obtains the real-time implementation of production logistics as data support of the synchronization decision to implement the global lean operation of the production logistics system.

This system utilizes the open and extensible AUTOM real-time manufacturing IOT framework, which is based on the ISA-95 international standard, to deploy the specific IOT devices in the key link of the production system execution environment in order to achieve the overall sensing of dynamic information. At the key point of production implementation field, such as workshop, warehouse, internal logistics vehicles, such as warehouse administrator, smart devices like RFID/QR code Multi-source label, Wi-Fi network environment, tablet PC and networking equipment are deployed to establish the IOT sensing environment. The production operators use the PDA to collect the information of material and equipment for on-site lean control. The forklift drivers obtain the accurate location information through the tablet PC to realize the lean control of vehicle route. Figure 7 shows the IOT devices used by the main roles of the executive layer and the data information feedback to the decision-makers. 
Fig. 7

IoT environment deployment

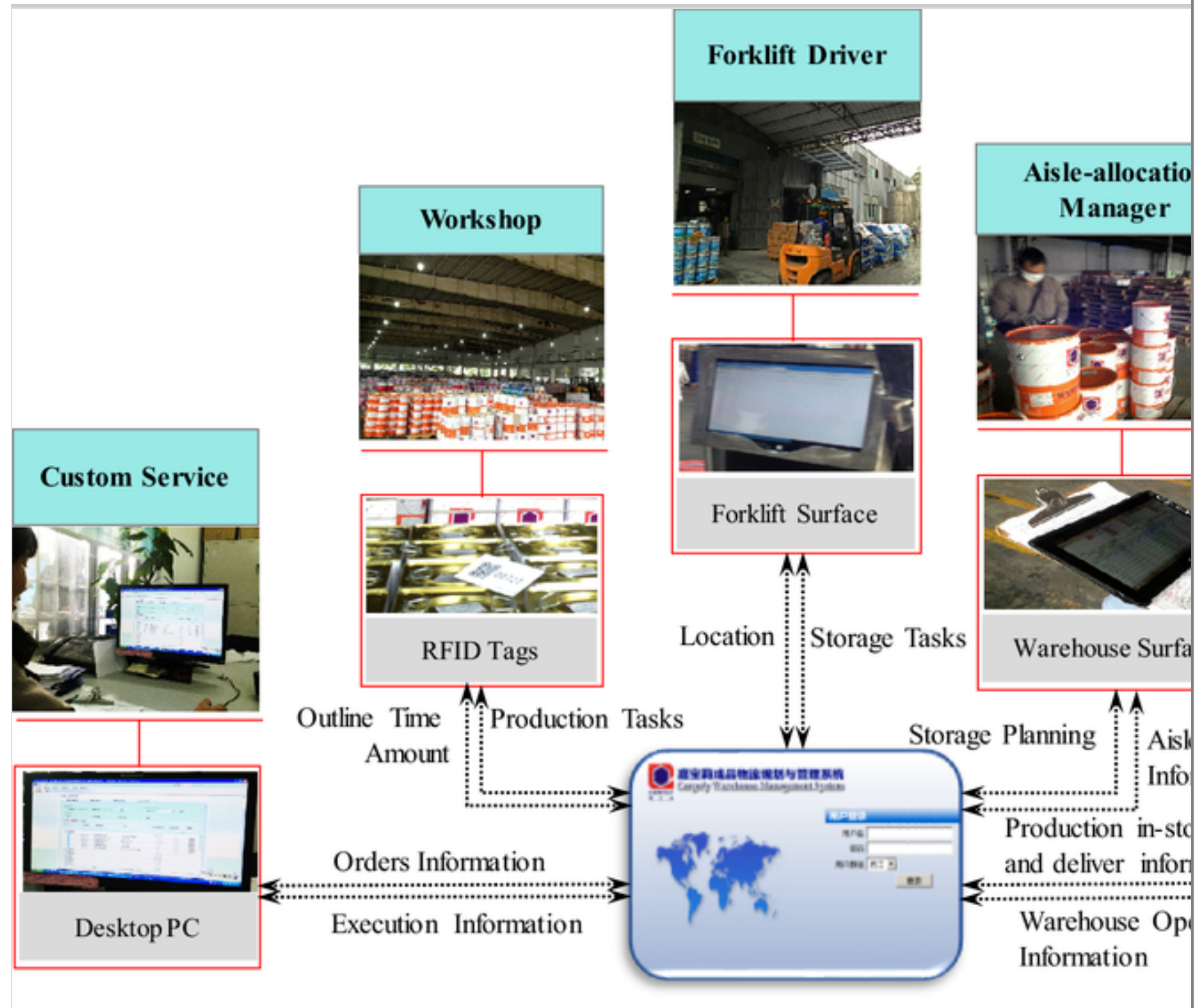

\subsection{The way using L-OSCT in this system}

\subsubsection{Formulation of theoretical optimum-state}

Through the IoT smart sensing devices, the system obtains real-time information of execution layer, including product offline time, forklift transportation time, storage location allocation, and delivery time and so on for accurate scheduling of personnel, materials, and equipment and warehouse resources to develop the global optimal production and storage allocation plan.

\subsubsection{Formulation of practical optimum-state}


When the actual state of the production logistics process deviates from the theoretical lean due to the dynamic interruption (such as emergency orders, machine failure), the system develops the newly optimal production plan and warehousing plan, which is the practical optimum-state, according to the real operation situation of each subsystem. For example, in the case of the allocation process of warehouse location, the two-stage dynamic lean control mechanism with pre-planning and dynamic programming is adopted. The warehouse operator makes the warehousing plan one day in advance. When the dynamics occur, the warehouse operator adjusts the storage allocation decision in accordance with the real state of the warehouse considering the orders schedule, aisle occupancy information and so on. For storage allocation rules, the rulebased algorithm and GA storage allocation rules are adopted to develop the lean warehousing planning, so the dynamic planning is consistent with the situation of the optimal actual theoretical decision.

\subsection{System implementation and deployment}

\subsubsection{System implementation}

The operation environment of the system is as follows:

- System architecture: B/S architecture, $\mathrm{C} / \mathrm{S}$ architecture (PDA)

- Development languages and platforms: C\#, Microsoft Visual Studio

- Database: Oracle $11 \mathrm{~g}$

- Operating environment: Windows 7, Windows Mobile

\subsubsection{System deployment}

The system is divided into six modules: intelligent PDA execution module, forklift loading module, warehouse management module, vehicle registration module, basic data management module and report management module. The main operation process is: order receiving, release production tasks, Workshop Scheduling, finished product offline binding, delivery tasks assigning, storage allocation, finished warehousing, finished goods allocation, delivery dispatching, finished delivery. Each process and its corresponding operation interface are shown in Fig. 8.

\section{Fig. 8}


Function flow and interface diagram

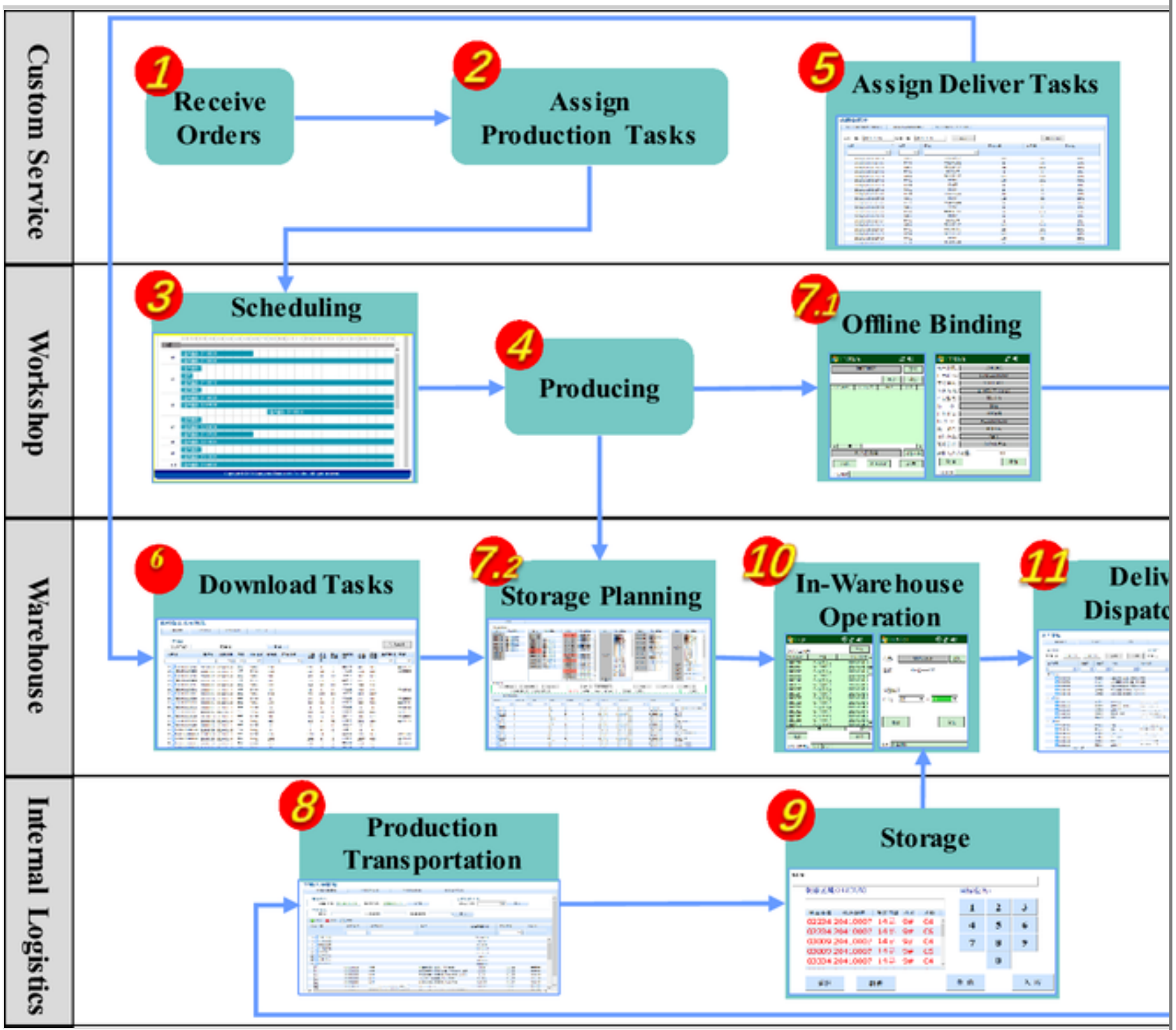

\subsection{Efficiency analysis}

A set of real data obtained from the system is used for analysis via MATLAB. The three important lean targets, including maximum makespan, aisle occupancy quantity and the total production cost, are analyzed and the efficiency of using the system is shown.

Firstly, we analyze the effect comparison of applying L-OSCT separately in warehouse and workshop. When the dynamic of new orders occurs, the effect of L-OSCT by comparing the practical lean curve of each department with the theoretical lean curve generated by utilizing single-unit lean control (SLC) and multi-unit lean control (MLC) separately can be verified. On the other hand, in case of the dynamic level (DL) changing, the control effect of SLC and MLC will be compared. 
As shown in Fig. 9, assume that the customer demand occurs at different time during production $(T=0,10,20,30)$ and the dynamic demand (DD) has different size ( $D D=40,120,200)$ which stand for different dynamic levels (from 1 to 3). In horizontal perspective, the practical lean data and actual data of the total cost, aisle occupancy and the maximum completion time under the same dynamic are given. The worse boundary lean curve is used for the theoretical lean curve. In the vertical perspective, the figure shows the different system performance by utilizing the single-unit lean control and multi-unit lean control under different dynamic level.

Fig. 9

Simulation result analysis

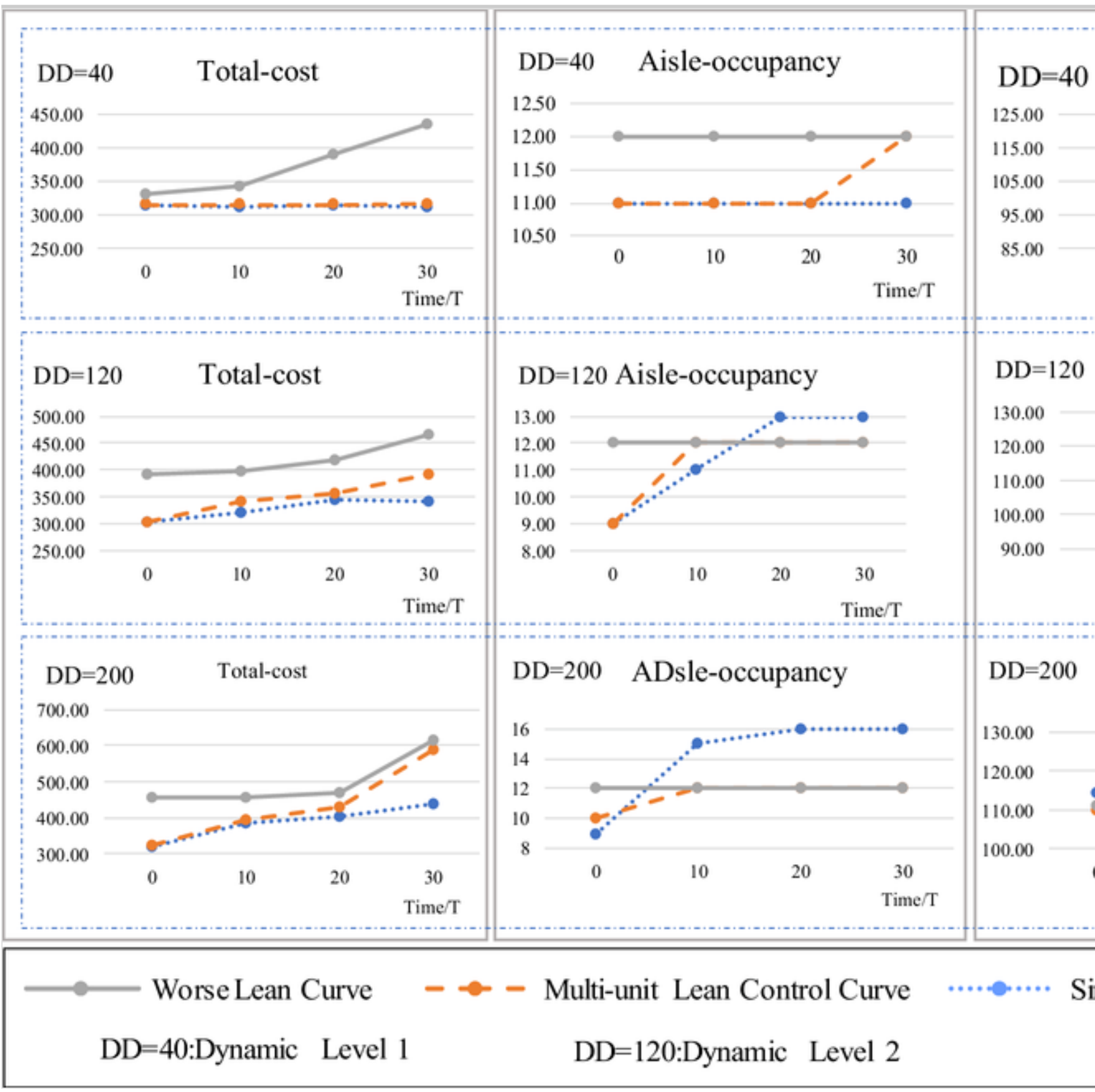


The Following observations can be obtained from Fig. 9:

1. When the dynamic occurs, the figure shows that the practical lean curve are always under the theoretical lean curves which indicates that performance of the production and warehouse departments and further the whole company achieves a higher level of lean.

2. For low-level dynamics the SCL is more effective to decrease the dynamics impact. Otherwise, the MCL is better.

In addition to the analysis of model data also shows that L-OSCT has high applicability in terms of operational efficiency in comparison to company statistics. Compared to the operation data captured from the warehouse department, the order linkage efficiency is increased by $30 \%$ and location occupying time decreased by $20 \%$ after using this system, besides, there have been no warehouse explosion phenomenon.

\section{Conclusion}

The emergence and subsequent popularization of lean has been one of the most significant developments in the history of operations management. However, there is a lack of systematic theory on the control framework underlying lean production. In response, several major LP methods and tools were redefined from the perspective of process control in this study. Then the theory of optimum-state control was introduced and its application to dynamic process control in industrial networking system exemplified. This control theory was then used to proposes a lean-oriented optimum state control (L-OSCT) theory. Using a IoT driven synchronization method, L-OSCT achieves the synchronization of the system optimum-state control for a large-scale production system with multi-workshop/plant in dynamic production environments. Finally, a case example of our new L-OSCT approach is provided.

Based on this research, we will focus on the following topic as the further research: The quantitative model of L-OSCT, and on the quantitative study of the mechanism of OSCT will be focused; The relationship between the local lean and the global lean will be find out; Analysis of the approach of decomposition of multidimensional optimum-state.

Publisher's Note 
Springer Nature remains neutral with regard to jurisdictional claims in published maps and institutional affiliations.

\section{Acknowledgements}

This work was supported by the National Natural Science Foundation of China (51475095)(51475095, 51875251), Natural Science Foundation of Guangdong Province (2016A030311041, 2017A030313401), 2015 Guangdong Special Support Scheme $(2014$ TQ01 × 706) $(2014$ TQ01X706), the National Ministry of Education "Blue Fire Plan" (Huizhou) Industry-Academia-Research Joint Innovation Fund (2018-2021) and the Fundamental Research Funds for the Central Universities (11618401).

\section{References}

Agus A, Shukri Hajinoor M (2012) Lean production supply chain management as driver towards enhancing product quality and business performance: case study of manufacturing companies in. Malays Int J Qual Reliab Manag 29:92-121

Azadeh A, Yazdanparast R, Zadeh SA, Zadeh AE (2017) Performance optimization of integrated resilience engineering and lean production principles. Expert Syst Appl 84:155-170

Bordel B, Alcarria R, Martín D, Robles T, de Rivera DS (2017) Selfconfiguration in humanized cyber-physical systems. J Ambient Intell Humaniz Comput 8:485-496. https://doi.org/10.1007/s12652-016-0410-3

Brewer A, Sloan N, Landers TL (1999) Intelligent tracking in manufacturing. J Intell Manuf 10:245-250

Chongwatpol J, Sharda R (2013) RFID-enabled track and traceability in jobshop scheduling environment European. J Oper Res 227:453-463

Chow HK, Choy KL, Lee W, Lau K (2006) Design of a RFID case-based resource management system for warehouse operations. Expert Syst Appl $30: 561-576$

Deshmukh G, Patil CR, Deshmukh MG (2017) Manufacturing industry performance based on lean production principles. In: Nascent Technologies in Engineering (ICNTE), 2017 International Conference on. IEEE, pp 1-6 
Dombrowski U, Mielke T, Engel C (2012) Knowledge management in lean production systems. Procedia Cirp 3:436-441

Fatorachian H, Kazemi H (2018) A critical investigation of industry 4.0 in manufacturing: theoretical. Oper Framew Prod Plan Control 29:633-644. https://doi.org/10.1080/09537287.2018.1424960

Fogliatto FS, Da Silveira GJ, Borenstein D (2012) The mass customization decade: an updated review of the literature. Int J Prod Econ 138:14-25

Herron C, Braiden PM (2006) A methodology for developing sustainable quantifiable productivity improvement in manufacturing companies. Int $\mathrm{J}$ Prod Econ 104:143-153

Holweg M (2007) The genealogy of lean production. J Oper Manag 25:420437

Huang GQ, Zhang Y, Jiang P (2008) RFID-based wireless manufacturing for real-time management of job shop WIP inventories The International. J Adv Manuf Technol 36:752-764

Huang GQ, Qu T, Fang MJ, Bramley AN (2011) RFID-enabled gateway product service system for collaborative manufacturing alliances CIRP. Ann Manuf Technol 60:465-468

Huang GQ, Qu T, Zhang Y, Yang H (2012) RFID-enabled product-service system for automotive part and accessory manufacturing alliances. Int J Prod Res 50:3821-3840

Jasti NVK, Kodali R (2016) Development of a framework for lean production system: an integrative approach proceedings of the institution of mechanical engineers, part B. J Eng Manuf 230:136-156

Li H-X, Si H (2017) Control for Intelligent Manufacturing. A Multiscale Chall Eng 3:608-615. https://doi.org/10.1016/J.ENG.2017.05.016

Marodin GA, Saurin TA (2013) Implementing lean production systems: research areas and opportunities for future studies International. J Prod Res $51: 6663-6680$

McLachlin R (1997) Management initiatives and just-in-time manufacturing. J Oper Manag 15:271-292 
Ning T, Huang M, Liang X, Jin H (2016) A novel dynamic scheduling strategy for solving flexible job-shop problems. J Ambient Intell Humaniz Comput 7:721-729. https://doi.org/10.1007/s12652-016-0370-7

Oh R, Park JA (2008) Development of active monitoring system for intelligent RFID logistics processing environment. In: Advanced language processing and Web information technology, 2008. ALPIT'08. International conference on IEEE, pp 358-361

Ohno T (1988) Toyota production system: beyond large-scale production. CRC Press, Boca Raton

Olivella J, Cuatrecasas L, Gavilan N (2008) Work organisation practices for lean production. J Manuf Technol Manag 19:798-811

Patil R, Avittathur B, Shah J (2010) Supply chain strategies based on recourse model for very short life cycle products International. J Prod Econ 128:3-10

Powell D, Skjelstad L (2012) RFID for the extended lean enterprise International. J Lean Six Sigma 3:172-186

Qi Q, Tao F (2018) Digital twin and big data towards smart manufacturing and industry 4.0: 360 degree. Comparison IEEE Access 6:3585-3593. https://doi.org/10.1109/ACCESS.2018.2793265

Qu T et al (2012a) RFID-enabled just-in-time logistics management system for 'SHIP'-supply Hub in industrial park CIE42 Proceedings

Qu T, Yang H, Huang GQ, Zhang Y, Luo H, Qin W (2012b) A case of implementing RFID-based real-time shop-floor material management for household electrical appliance manufacturers. J Intell Manuf 23:2343-2356

Qu T, Zhang L, Huang Z, Dai Q, Chen X, Huang GQ, Luo H RFID-enabled smart assembly workshop management system. In: Networking, Sensing and Control (ICNSC), 2013 10th IEEE International Conference on (2013) IEEE, pp 895-900

Qu T, Lei S, Wang Z, Nie D, Chen X, Huang GQ (2016) IoT-based real-time production logistics synchronization system under smart cloud manufacturing. Int J Adv Manuf Technol 84:147-164 
Qu T, Pan Y, Liu X, Kang K, Li C, Thurer M, Huang GQ (2017a) Internet of Things-based real-time production logistics synchronization mechanism and method toward customer order dynamics. Trans Inst Measur Control 39:429445

Qu T, Thürer M, Wang J, Wang Z, Fu H, Li C, Huang GQ (2017b) System dynamics analysis for an Internet-of-Things-enabled production logistics system. Int J Prod Res 55:2622-2649

Qu T, Zhang K, Yan M, Guo H, Huang GQ, Li C, Li X (2018) Synchronized decision-making and control method for opti-state execution of dynamic production systems with internet of things. J Mech Eng (Accepted) AQ2

Riezebos J, Klingenberg W, Hicks C (2009) Lean production and information technology: connection or contradiction? Comput Ind 60:237-247

Saucedo-Martínez JA, Pérez-Lara M, Marmolejo-Saucedo JA, Salais-Fierro TE, Vasant P (2018) Industry 4.0 framework for management and operations: a review. J Ambient Intell Humaniz Comput 9:789-801. https://doi.org/10.1007/s12652-017-0533-1

Shah R, Ward PT (2003) Lean manufacturing: context, practice bundles, and performance. J Oper Manag 21:129-149

Shah R, Ward PT (2007) Defining and developing measures of lean production. J Oper Manag 25:785-805

Sim KL, Rogers JW (2008) Implementing lean production systems: barriers to change. Manag Res News 32:37-49

Sugimori Y, Kusunoki K, Cho F, Uchikawa S (1977) Toyota production system and kanban system materialization of just-in-time and respect-forhuman system. Int J Prod Res 15:553-564

Tao F, Qi Q (2017) New IT driven service-oriented smart manufacturing: framework and characteristics IEEE transactions on systems, man, and cybernetics. Systems. https://doi.org/10.1109/TSMC.2017.2723764

Tao F, Zhao D, Hu Y, Zhou Z (2008) Resource service composition and its optimal-selection based on particle swarm optimization in manufacturing grid 
system. IEEE Trans Ind Inf 4:315-327.

https://doi.org/10.1109/TII.2008.2009533

Tao F, Cheng J, Cheng Y, Gu S, Zheng T, Yang H (2017) SDMSim: a manufacturing service supply-demand matching simulator under cloud environment. Robot Comput Integr Manuf 45:34-46.

https://doi.org/10.1016/j.rcim.2016.07.001

Tao F, Cheng J, Qi Q (2018) IIHub: an industrial internet-of-things hub toward smart manufacturing based on cyber-physical system. IEEE Trans Industr Inf 14:2271-2280. https://doi.org/10.1109/TII.2017.2759178

Wang M, Qu T, Zhong RY, Dai Q, Zhang X, He J (2012) A radio frequency identification-enabled real-time manufacturing execution system for one-ofa-kind production manufacturing: a case study in mould industry. Int $\mathrm{J}$ Comput Integr Manuf 25:20-34

Zhang L (2008) Lean production and labor controls in the Chinese automobile industry in an age of globalization International. Labor WorkingClass History 73:24-44

Zhang Y, Qu T, Ho O, Huang GQ (2011a) Real-time work-in-progress management for smart object-enabled ubiquitous shop-floor environment. Int J Comput Integr Manuf 24:431-445

Zhang Y, Qu T, Ho OK, Huang GQ (2011b) Agent-based smart gateway for RFID-enabled real-time wireless manufacturing. Int J Prod Res 49:13371352

Zhang Y, Xu J, Sun S, Yang T (2015) Real-time information driven intelligent navigation method of assembly station in unpaced lines. Comput Ind Eng $84: 91-100$

Zhang Y, Qian C, Lv J, Liu Y (2017) Agent and cyber-physical system based self-organizing and self-adaptive intelligent shopfloor. IEEE Trans Ind Inf 13:737-747. https://doi.org/10.1109/TII.2016.2618892

Zhang Y, Guo Z, Lv J, Liu Y (2018a) A Framework for smart productionlogistics systems based on CPS and industrial IoT. IEEE Trans Ind Inf. https://doi.org/10.1109/TII.2018.2845683 
Zhang Y, Zhu Z, Lv J (2018b) CPS-based smart control model for shopfloor material handling. IEEE Trans Industr Inf 14:1764-1775.

https://doi.org/10.1109/TII.2017.2759319 